\title{
Empagliflozin in paroxysmal or persistent atrial fibrillation: a possible antiarrhythmic effect
}

\author{
Fazio $G^{*}$ and Schirò $P$ \\ Casa di Cure Triolo Zancla - Piazza Fonderia 23 - 90100 - Palermo, Italy
}

\begin{abstract}
Background: Many studies have highlighted an increased risk of developing atrial fibrillation (AF) in diabetic patients. The SGLT2 inhibitors, in addition to glycaemic control, have shown pleiotropic effects on the cardiovascular system, proving to be particularly advantageous in patients with heart failure. In this study we evaluated a group of patients with paroxysmal $\mathrm{AF}(\mathrm{PAF})$ or persistent $\mathrm{AF}(\mathrm{PeAF})$ affected by type 2 diabetes and treated with empagliflozin.
\end{abstract}

Methods: This is a case-crossover study obtained from our database of patients affected by PAF or PeAF. We extracted 23 consecutive subjects ICD wearers and affected by diabetes that started treatment with empagliflozin during the follow-up.

Results: All enrolled patients were ICD wearers because at high risk of sudden cardiac death and received amiodarone; empagliflozin was added during follow-up for the treatment of diabetes. We evaluated the recurrence of PAF and the days free from AF one year before and one year after starting treatment with empagliflozin. The recurrence of AF was significantly lower after starting empagliflozin than before the treatment (p: 0.001).

Conclusion: Our data suggest that empagliflozin is able to reduce the recurrences of AF in diabetic patients with PAF or PeAF. Further studies might demonstrate an antiarrhythmic effect of empagliflozin and change the therapeutic approach in subjects at high risk of AF.

\begin{abstract}
Abbreviations: CHD: Chronic heart failure; HF: Herat failure; PAF: paroximal atriale fibrillation; AF: Atrial fibrillation; SGLT: Sodium/glucose co-trasporter 2.
\end{abstract}

\section{Introduction}

Traditionally, diabetes mellitus has been associated with coronary heart disease (CHD), but the possible correlation with arrhythmic diseases is a more recent concern. Over the last decade many studies have highlighted the association between diabetes mellitus and increased risk of developing atrial fibrillation (AF) relative to patients without diabetes [1-7]. The SGLT2 inhibitors, in addition to the hypoglycaemic effect, have shown pleiotropic effects on the cardiovascular system, proving to be particularly advantageous in patients with heart failure. The post-hoc analysis of the DECLARE-TIMI 58 study conducted by Zelniker and colleagues, recently published in Circulation, for the first time demonstrated a favourable effect of treatment with the SGLT2 inhibitor dapagliflozin on the incidence of FA and FLA in patients with type 2 diabetes, independently of previous history of FA / FLA, atherosclerotic disease or heart failure [8]. The mechanisms underlying this effect are not yet well known and are probably multiple: from the natriuretic, diuretic and hemodynamic effect of these drugs that could prevent atrial dilation and cardiac remodelling, to the effects on blood pressure, body weight, oxidative stress and reduction of epicardial fat.

In this study we analysed retrospectively our experience in a cohort of diabetic patients with paroxysmal AF (PAF) or persistent AF (PeAF) in treatment with empagliflozin for diabetes.

\section{Methods}

Data of the present case-crossover study were retrospectively obtained from our database of patients affected by PAF or PeAF: from
2540 patients followed for AF by our cardiology unit, we extracted 23 consecutive subjects ICD wearers and affected by diabetes that started treatmentwith empagliflozin during the follow-up. The mean age of this study group was $72,6 \pm 6$ years. PAF were diagnosedin $88,5 \%$ subjects whereas PeAF in the remaining $12,5 \%$. All enrolled patients were ICD wearers and received amiodarone. The ICD was implanted due to high cardiovascular risk when the FE was $\leq 35 \%$ and/or the QT was elongated; empagliflozin was added during follow-up for the treatment of diabetes (Table 1).

All patients are monitored at least twice per year, through a clinical examination, an electrocardiogram and blood routine examinations. Given the need for renewal of the therapeutic plan, all patients treated with empaglifozin must return at least once six months to the prescriber centre and therefore none of the patients who continued the treatment was lost at the follow-up. No patient discontinued the treatment.

Patient notes were reviewed, and the following data recorded: age, sex, indication for empaglifozin, duration of treatment, concomitant therapy, creatinine and haemoglobin level before and after treatment.

The present project is an observational retrospective study and so it is not subject to the directive 2001/20/CE of the European Parliament regulating the application of a good clinical practice during clinical

${ }^{\star}$ Correspondence to: Giovanni Fazio, MD, PhD, FESC, FEACVI, Casa di Cure Triolo Zancla - Piazza Fonderia 23 - 90100 - Palermo, Italy, E-mail: Faziogiova@gmail.com

Key words: empaglifozin, atrial fibrillation, recurrences, inflammation

Received: December 24, 2020; Accepted: January 15, 2021; Published: January 19,2021 
experimentation (Art. 1 comma 1; Art 2/c) and so it was not necessary to require an Ethics Committee ruling.

\section{Results}

We evaluated the recurrence of PAF and the days free from AF one year before and one year after starting treatment with empagliflozin. The recurrence of AF was lower after starting empagliflozin than before the treatment. We reported statistical differences both in the number of episodes of recurrence and on days without arrhythmia (p: 0.001 and p: 0.04) (Table 2).

\section{Discussion}

Atrial fibrillation is the most frequent sustained cardiac arrhythmia, associated with a significant increase in the risk of thromboembolic events and mortality. From 50 years onwards, the prevalence of AF doubles every 10 years, reaching $10 \%$ after 80 years [9]. Treatment of AF has as first aim the restoration and maintenance of the sinus rhythm. However, drugs used to date have limitations in terms of side and pro-arrhythmic effects, compared to a limited antiarrhythmic effect. Due to contraindications and possible risks, rhythm control in elderly patients with AF is an actionable goal only in selected patients, while in most subjects the only achievable objective remains control of frequency. The post-hoc analysis of the DECLARE-TIMI 58 study conducted by Zelniker and colleagues, published last January in Circulation, suggests first a possible role of a SGLT2 inhibitor in the prevention of AF [8]. In addition, many new antidiabetic agents

Table 1. Characteristics of patients

\begin{tabular}{|c|c|}
\hline No. of patients $[n(\%)]$ & 23 \\
\hline Age (years) [mean \pm SDI] & $72,7 \pm 6$ \\
\hline Male/female $[n(\%)]$ & $48 \% / 52 \%$ \\
\hline Creatinine (mg/dL) [mean \pm SD] & $1.2 \pm 1$ \\
\hline Months of anticoagulation therapy [mean & $24 \pm 2$ \\
\hline SD] & $88,50 \%$ \\
\hline Paroximal AF $[n(\%)]$ & $12,50 \%$ \\
\hline persistent AF $[n(\%)]$ & $83 \%$ \\
\hline Hypertension $[n(\%)]$ & $23 \%$ \\
\hline Hypertensive Cardiomyopathy & $100 \%$ \\
\hline Diabetes mellitus $[n(\%)]$ & $13000 \%$ \\
\hline Heart failure $[n(\%)]$ & $8,30 \%$ \\
\hline Previous Stroke/TlA $[n(\%)]$ & $60,00 \%$ \\
\hline Ischemic cardiomyopathy $[n(\%)]$ & $36,25 \%$ \\
\hline$\beta$-Blockwera $[n(\%)]$ & $100,00 \%$ \\
\hline Amiodaron $[n(\%)]$ & $100,00 \%$ \\
\hline empaglifozin & $23,00 \%$ \\
\hline insulin & $93,00 \%$ \\
\hline metformin & $4.02 \pm 1.5$ \\
\hline CHA2DS2-VASc (mean \pm SD) & $2.50 \pm 0.7$ \\
\hline HAS-BLED score (mean \pm SD) & \\
\hline
\end{tabular}

Table 2. Results of the analysis

\begin{tabular}{|c|c|c|c|}
\hline & $\begin{array}{c}\text { Before } \\
\text { empaglifozin }\end{array}$ & $\begin{array}{c}\text { After } \\
\text { empaglifozin }\end{array}$ & P \\
\hline $\begin{array}{c}\text { Recurrences of paroxysmal at rial } \\
\text { fibrillation/patients year }\end{array}$ & 6,5 & 2,3 & 0,001 \\
\hline Days free from AF/patient year & 244 & 303 & 0,04 \\
\hline Cardiovascular death & $3 \%$ & $3100 \%$ & NS \\
\hline TIA & $6,25 \%$ & $1,2 \%$ & 0,0046 \\
\hline Stroke & $0 \%$ & $0,02 \%$ & NS \\
\hline Systemic embolism & $0 \%$ & $0,001 \%$ & NS \\
\hline Pulmonary embolism & $0 \%$ & $0,001 \%$ & NS \\
\hline
\end{tabular}

have been recently demonstrating possible therapeutic benefits even in patients without diabetes. This is what emerges from the phase III trials DAPA-HF and EMPEROR-Reduced where the effect of dapagliflozin and empagliflozin, respectively, on HF, in addition to the standard of care, has been investigated in adult patients with reduced ejection fraction with and without type 2 diabetes [10,11]. Our clinical experience with empagliflozin suggests that this molecule may also exertan antiarrhythmic effect on the heart, in a similar way to that observed with dapagliflozin in DECLARE-TIMI 58. Empagliflozin is an oral antidiabetic drug that acts by inhibiting the action of the sodium-glucose cotransporter 2 at the level of the renal tubule with a greater selectivity than dapagliflozin. Recent studies on the cardiovascular safety profile of the drug have shown that empagliflozin reduces mortality and hospitalization for heart failure (HF) compared to placebo, but to date it is not clear which mechanisms are responsible for these effects [12-15]. Data obtained from our small-scale study indicate that, in addition to already known effects, empagliflozin might exert an antiarrhythmic effect. The mechanisms underlying the possible protective effect of empagliflozin on FA are still unknown. We therefore hypothesize that SGLT2 inhibitors modulate various risk factors for FA such as myocardial remodelling, blood pressure, body weight, inflammation, oxidative stress and adrenergic hyperactivity [16-19]. Further studies should investigate the role of SGLT inhibitors in the prevention and treatment of FA thus unveiling potential new treatment of AF, especially in people with HF.

\section{Conclusion}

Our data might suggest that empagliflozin is able to reduce the recurrences of AF in diabetic patients with PAF or PeAF. This smallscale case-crossover study aims to encourage research of clinical evidence, through specially designed studies, in order to optimize the use of available antidiabetic drugs for the prevention and treatment of cardiovascular diseases in patients at high cardio-metabolic risk.

\section{References}

1. Benjamin EJ, Levy D, Vaziri SM (1994) Independent risk factors for atrial fibrillation in a population-based cohort. The Framingham Heart Study. JAMA 271: 840-844. [Crossref]

2. Dublin S, Glazer NL, Smith NL (2010) Diabetes mellitus, glycemic control, and risk of atrial fibrillation. J Gen Intern Med 25: 853-858. [Crossref]

3. Huxley RR, Filion KB, Konety S, Alonso A (2011) Meta-analysis of cohort and casecontrol studies of type 2 diabetes mellitus and risk of atrial fibrillation. Am J Cardiol 108: 56-62. [Crossref]

4. Yamagishi SI, Matsui T, Nakamura K (2008) Possible molecular mechanisms by which angiotensin II type 1 receptor blockers (ARBs) prevent the development of atrial fibrillation in insulin resistant patients. Horm Metab Res 40: 640-644. [Crossref]

5. Ostgren CJ, Merlo J, Rastam L (2004) Hypertension and Diabetes Project. Atrial fibrillation and its association with type 2 diabetes and hypertension in a Swedish community. Diabetes Obes Metab 6: 367-374. [Crossref]

6. Dublin S, Glazer NL, Smith NL (2010) Diabetes mellitus, glycemic control, and risk of atrial fibrillation. J Gen Intern Med 25: 853-858. [Crossref]

7. Fangel MV, Nielsen PB, Kristensen JK (2019) Glycemic status and thromboembolic risk in patients with atrial fibrillation and type 2 diabetes mellitus: A Danish cohort study. Circ Arrhythm Electrophysiol 12: e007030. [Crossref]

8. Zelniker TA, Bonaca MP, Furtado RHM (2020) Effect of dapagliflozin on atrial fibrillation in patients with type 2 diabetes mellitus: Insights From the DECLARETIMI 58 Trial. Circulation 141: 1227-1234. [Crossref]

9. Go AS, Hylek EM, Phillips KA (2001) Prevalence of diagnosed atrial fibrillation in adults: national implications for rhythm management and stroke prevention: The AnTicoagulation and Risk Factors in Atrial Fibrillation (ATRIA) Study. JAMA 285 2370-2375. [Crossref] 
10. McMurray JJV, Solomon SD, Inzucchi SE (1995) Dapagliflozin in patients with heart failure and reduced ejection fraction. $N$ Engl J Med 381: 1995-2008.

11. Petrie MC, Verma S, Docherty KF (2020) Effect of dapagliflozin on worsening heart failure and cardiovascular death in patients with heart failure with and without diabetes. JAMA 323: 1353-1368. [Crossref]

12. Zinman B, Wanner C, Lachin JM (2015) Empagliflozin, cardiovascular outcomes, and mortality in type 2 diabetes. $N$ Engl J Med 373: 2117-2128. [Crossref]

13. Fitchett D, Zinman B, Wanner C (2016) Heart failure outcomes with empagliflozin in patients with type 2 diabetes at high cardiovascular risk: results of the EMPA-REG OUTCOME® trial. Eur Heart J 37: 1526-1534. [Crossref]

14. Fitchett D, Inzucchi SE, Cannon CP (2019) Empagliflozin reduced mortality and hospitalization for heart failure across the spectrum of cardiovascular risk in the EMPA-REG OUTCOME trial. Circulation 139: 1384-1395. [Crossref]
15. Furtado RHM, Bonaca MP, Raz I (2019) Dapagliflozin and cardiovascular outcomes in patients with type 2 diabetes mellitus and previous myocardial infarction. Circulation 139: 2516-2527. [Crossref]

16. Santos-Gallego CG, Garcia-Ropero A, Mancini D (2019) Rationale and design of the EMPA-TROPISM Trial (ATRU-4): Are the "Cardiac Benefits" of Empagliflozin Independent of its Hypoglycemic Activity? Cardiovasc Drugs Ther 33: 87-95. [Crossref]

17. Natali A, Nesti L, Fabiani I, Calogero E, Di Bello V (2017) Impact of empagliflozin on subclinical left ventricular dysfunctions and on the mechanisms involved in myocardial disease progression in type 2 diabetes: rationale and design of the EMPA-HEART trial. Cardiovasc Diabetol 16: 130. [Crossref]

18. Li C, Zhang J, Xue M (2019) SGLT2 inhibition with empagliflozin attenuates myocardial oxidative stress and fibrosis in diabetic mice heart. Cardiovasc Diabetol 18: 15. [Crossref]

19. Herat LY, Magno AL, Rudnicka C (2020) SGLT2 Inhibitor-Induced sympathoinhibition: A novel mechanism for cardiorenal protection. JACC Basic Transl Sci 5: 169-179. [Crossref]

Copyright: $(\mathbb{O} 2021$ Fazio G. This is an open-access article distributed under the terms of the Creative Commons Attribution License, which permits unrestricted use, distribution, and reproduction in any medium, provided the original author and source are credited. 